\title{
Erratum to: Impact de l'assurance maladie sur le coût financier direct de la prise en charge medicale de la cirrhose au centre hospitalier et universitaire campus de Lomé, Togo
}

Erratum to: Impact of the health insurance on the financial direct cost of the cirrhosis medical management at campus teaching hospital of Lomé, Togo

L.M. Lawson-Ananissoh · O. Bouglouga · A. Bagny · L. Kaaga · R. El-Hadj Yakoubou $\cdot$ D. Redah

(C) Lavoisier SAS 2014

Erratum to: $10.1007 / \mathrm{s} 12558-014-0573-9$

Cet article du Journal africain d'hépato-gastroentérologie s'est vu malencontreusement publié dans la liste des articles Online First d'une autre revue, à la suite d'une erreur d'identification numérique. L'éditeur présente ses excuses aux auteurs et aux lecteurs pour ce désagrément. 